\title{
Comparative analysis of probabilistic neural network, radial basis function, and feed- forward neural network for fault classification in power distribution systems
}

\begin{abstract}
This article presents a classification methodology based on probabilistic neural networks. To automatically select the training data and obtain the performance evaluation results, the ñKfoldò cross-validation method is used. Then, the probabilistic neural network is compared with the feed-forward neural network and the radial basis function network. The goal is to propose a classifier that is capable of recognizing 11 classes of three-phase distribution system faults to solve the complex fault (three-phase short-circuit) classification problem for reducing the multiple-estimation problem to estimate the fault location in radial distribution systems. The data for the fault classifier is produced by DigSilent Power Factory, Integrated Power System Analysis Software on an IEEE 13-node test feeder. A selection of features or descriptors obtained from voltages and currents measured in the substation are analyzed and used as input of the probabilistic neural network classifier. It is shown that the probabilistic neural network approach can provide a fast and precise operation for various faults. The simulation results also show that the proposed model can successfully be used as an effective tool for solving complicated classification problems.
\end{abstract}

Keyword: Fault classification; Feed-forward neural network; K -fold cross-validation; Power distribution systems; Probabilistic neural networks; Radial basis function network 